children and adolescents with Tourette's disorder. Am I Psychiatry August 1997;154:1057-1062). (Reprints: Dr Floyd R Sallee, Institute of Psychiatry, 67 Presidents St, Room 246, Charleston, SC 29425).

COMMENT. Pimozide is superior to haloperidol in therapeutic efficacy in children and adolescents with Tourette syndrome, and the incidence of extrapyramidal side effects is significantly lower. Only severe cases with a Tourette Symptom Global Scale score greater than 20 were included in the above trial, and exclusion criteria included: serious medical illness, abnormal ECG, use of other concurrent medication for asthma or ADHD (eg. theophylline, stimulants).

\title{
MECHANISM AND SIGNIFICANCE OF MIRROR MOVEMENTS
}

Neurophysiological studies, including EMG and focal magnetic brain stimulation, were used to evaluate possible mechanisms for mirror movements in 14 male patients, aged 16-60 years, with X-linked Kallmann's syndrome compared to controls at the Department of Physiology, University College London, UK. During EMG recording from the first dorsal interosseous muscle (1DI) with voluntary abduction of an index finger, mirror EMG activity was recorded simultaneously from the contralateral 1DI. Focal magnetic stimulation of the hand area of the motor cortex revealed inter- and intrasubject differences in the ratio of ipsilaterally to contralaterally projecting corticospinal axons. Multi-unit EMGs during simultaneous voluntary activation of distal upper limb muscles showed a central peak in the cross-correlograms, indicating a common drive to left and right homologous motor neuron pools. A synchronous activation of intermingled ipsilateral and contralateral corticospinal neurons could explain this common drive. Long latency transcortical components of a cutaneomuscular reflex recorded from the 1DI following digital nerve stimulation could be recorded simultaneously from the $1 \mathrm{DI}$ of the non-stimulated side. Activity in a novel ipsilateral corticospinal tract is proposed as the mechanism for pathological mirror movements in Xlinked Kallmann's syndrome. (Mayston MJ, Harrison LM, Quinton R et al. Mirror movements in X-linked Kallmann's syndrome. 1. A neurophysiological study. Brain July 1997;120:1199-1216). (Respond: Dr Linda M Harrison, Department of Physiology, University College London, Gower Street, London WC1E 6BT, UK).

COMMENT. The X-linked form of Kallmann's syndrome (KS) is characterized by hypogonadotrophic hypogonadism, anosmia, unilateral renal agenesis, and mirror movements. An adolescent male of eunuchoid build, referred to the neurologist because of attention, coordination, and cognitive deficits, should be considered for olfactory tests and an MRI of the brain, for aplasia of the olfactory system. (see Progress in Pediatric Neurology II, PNB Publ, 1994;p309). The abnormal mirror movements observed in $85 \%$ of adult patients with KS are a normal and frequent finding in children under 7 years of age. The persistence of mirror movements, often observed in older children with ADHD, can be a sign of "minimal brain dysfunction," and an inability to inhibit activity in the ipsilateral corticospinal tract during rapid alternating movement of one hand. A defect or delay in myelination of callosal fibers has been suggested as the underlying pathology for persistent mirror movements. They are often present with callosal agenesis. In KS patients the defect may also extend to other axonal fibers in the frontal motor and olfactory systems, and involving a novel abnormal ipsilateral corticospinal tract.

In a further study using PET and regional cerebral blood flow, at the 
Wellcome Department of Cognitive Neurology, Queen Square, London, six right-handed male subjects with KS showed activation in the motor cortex both contralateral and ipsilateral to the voluntarily moved hand, although the contralateral activation was significantly stronger. Activation of the ipsilateral motor cortex in KS may be caused by sensory feedback from the involuntarily mirroring hand. (Krams M, Quinton R, Mayston MJ et al. Mirror movements in X-linked Kallmann's syndrome. II A PET study. Brain July 1997;120:1217-1228).

\section{HEADACHE DISORDERS}

\section{PSEUDOMIGRAINE PLEOCYTOSIS SYNDROME}

A series of 50 patients with a total of 164 episodes of pseudomigraine with temporary neurologic symptoms and CSF lymphocytic pleocytosis (PMP syndrome) is reported from the University Hospital Marques de Valdecilla, Santander, Spain. Onset was between 14 and 39 years, $68 \%$ in males. About one third had a past history of migraine, and one-quarter had a viral-like illness within 3 weeks of onset. A throbbing, bilateral headache lasting an average of 19 hours was associated with unilateral sensory symptoms ( $78 \%$ of episodes) of mean duration 5 hours, aphasic (66\%) and motor (56\%) symptoms, and visual symptoms in only $12 \%$ of episodes. Lymphocytic pleocytosis was 10 to 760 cells $/ \mathrm{mm} 3$ (mean, 199). CSF protein was increased. Viral studies were negative. EEG showed focal slowing. Angiography in 12 patients was normal except one showing localized vasculitis. An aseptic inflammation of leptomeningeal vasculature is suggested as a possible cause. (Gomez-Aranda F, Canadillas F, Marti-Masso JF, et al. Pseudomigraine with temporary neurological symptoms and lymphocytic pleocytosis. A report of 50 cases. Brain July 1997;120:11051113). (Respond: Dr Julio Pascual, Service of Neurology, University Hospital Marques de Valdecilla, 39008 Santander, Spain).

COMMENT. This appears to be the largest series of patients reported with the syndrome of transient headache and CSF lymphocytosis. A previous report of 7 patients and review of 33 cases in the literature, 13 in children and adolescents, were included in Ped Neur Briefs, Oct 1995 (see Progress in Pediatric Neurology III, 1997;p178). The differential diagnoses listed by the authors (Berg MJ, Williams LS) included Lyme neuroborreliosis, neurosyphilis, neurobrucellosis, neoplastic meningitis, HIV meningitis, hemiplegic migraine, seizures, Mollaret's meningitis, and a side effect of angiography. The syndrome is self limited, and a viral etiology appears plausible.

\section{ATTENTION DEFICIT AND LEARNING DISORDERS}

\section{LARGE CAUDATE NUCLEUS ON MRI IN ADHD}

MRI measurements of the head of the caudate nucleus correlated with neuropsychological deficits and behavioral problems in 11 adolescents with ADHD in a study at the University of Barcelona, Spain. The ADHD group had a larger right caudate nucleus and a trend toward a larger left caudate than a control group of 19 healthy subjects. Larger caudate nuclei in controls were associated with poorer performance on tests of attention and higher ratings on the Conners Teachers Rating Scale. A L $>$ R pattern of caudate asymmetry was present in the control group and a reverse pattern $(R>L)$ for the ADHD subjects. A bilateral dysfunction is suggested for ADHD, more pronounced on 